\title{
Post-traumatic Lung Embolization as a Bridge to Surgery in a Jockey Injured by a Fall from a Horse
}

\author{
Filippo Piacentino MD ${ }^{1,2}$, Christian Ossola MD ${ }^{1,2}$, Federico Fontana MD ${ }^{1,2}$, \\ Marco Curti MD ${ }^{1,2}$, Marta Duvia MD ${ }^{1,2}$, Giada Zorzetto MD ${ }^{1,2}$ \\ and Massimo Venturini Prof ${ }^{1,2}$ \\ 'Diagnostic and Interventional Radiology Department, Ospedale di Circolo, Varese, Italy \\ 2Insubria University, Varese, Italy
}

Keywords: Lung; Endovascular Embolization; Bleeding; Intervention Radiology; Trauma

\section{BACKGROUND}

The mortality of trauma with severe chest injury showing greater than 3 on the Abbreviated Injury Scale (AIS) is very high $-15.1 \%$ in all age groups and $28.4 \%$ in those 65 years or older [1]. Quick hemostasis and treatments with appropriate prioritization for injured organs are essential to rescue patients with polytrauma, especially severe truncal trauma with pulmonary contusion and massive hemorrhage.

Severe lung contusion can lead to massive hemothorax and severe tracheobronchial bleeding. Respiratory dysfunction can also be caused by blood flowing into the uninjured area from the pulmonary contusion.

\section{CASE PRESENTATION}

A 35-year-old woman with no past medical history was admitted to our emergency department following

\section{Corresponding author:}

Christian Ossola, Radiology Department, Ospedale di Circolo e Fondazione Macchi, padiglione monoblocco, Via Guicciardini 9, Varese - 21100, Italy.

Email: c.ossola7@gmail.com

(C) 2021 CC BY 4.0 - in cooperation with Depts. of Cardiothoracic/ Vascular Surgery, General Surgery and Anesthesia, Örebro University Hospital and Örebro University, Sweden a fall from a horse during a show-jumping competition. An initial evaluation of the site highlighted a severe non-penetrating thorax trauma due to direct compression by the animal.

Heli transported her to the emergency block, at which time her respiratory rate was 35 breaths/min, hemoglobin level was $12.6 \mathrm{~g} / \mathrm{dL}$ with a low blood oxygen saturation $(90 \%)$ and an acceptable blood pressure of $110 / 70 \mathrm{mmHg}$. A focused assessment sonography for trauma (FAST) revealed a hemothorax in the left side of the chest.

The contrast enhanced computed tomography (CT; Revolution Evo, General Electric, USA) performed in the emergency block demonstrated a massive left lung contusion of about $10 \times 6 \times 16 \mathrm{~cm}^{3}$, with important active contrast media extravasation in the lower lobe; multiple ribs fractures; and numerous fractures of transverse processes and dorsal vertebrae (Figure $1 a-c$ ).

After a multidisciplinary council, the trauma leader asked for a percutaneous embolization to achieve hemodynamic stability and to take time to possibly perform a lobectomy under better clinical conditions. The patient was thus moved to the angio-suite and a percutaneous right 5-F right common femoral vein sheath was managed, and digital subtraction angiography (DSA) confirmed active bleeding from the tributary arterial branch of the apical segment of the lower left lobe.

To confirm presence in the branch of the bleeding tributary pulmonary artery, a cone-beam CT was performed. Using a 2.7-F microcatheter (Progreat, Terumo, 


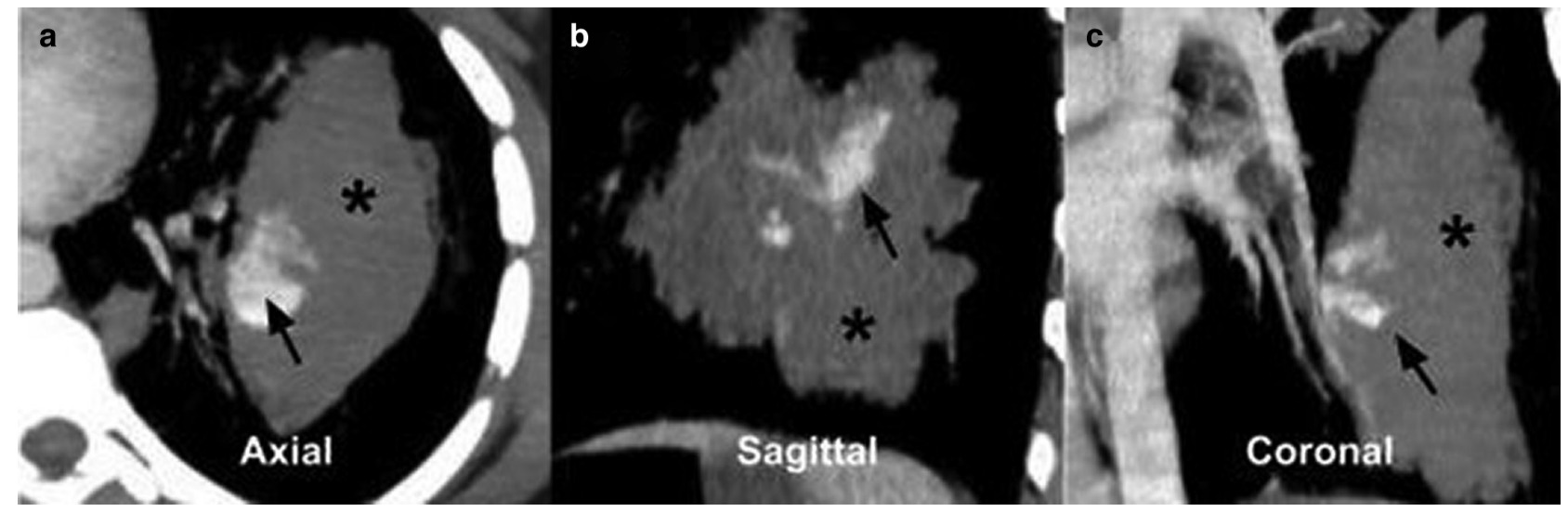

Figure 1 (a-c) A multiplanar maximum intensity projection shows the presence of active bleeding with massive left lung contusion (black asterisk) and contrast media extravasation in the lower lobe (black arrow).

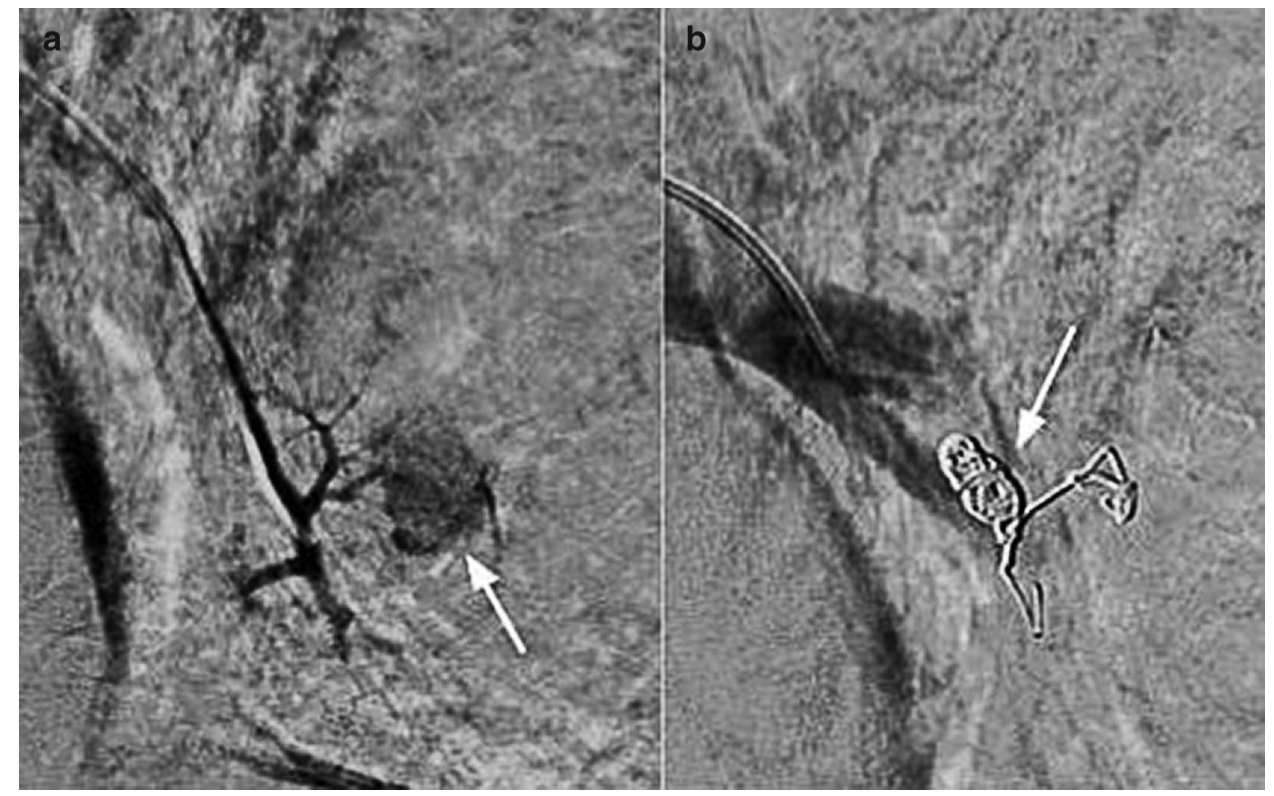

Figure 2 (a) Digital subtraction angiography (DSA) of the right pulmonary artery confirms massive active bleeding from the tributary arterial branch of the apical segment of the lower left lobe (white arrow). (b) A super-selective cannulation of the target arterial branch allows the deployment of micro-coils with endovascular hemostasis and complete resolution of bleeding.

Japan), the bleeding site was reached and three vascular occlusive micro-coils were deployed (Rubycoil soft, Penumbra Inc. Crossmed, USA).

A final DSA shows complete resolution of the bleeding (Figure $2 a, b)$.

The patient had a regular post-procedure course with hemodynamic stability; however, after $48 \mathrm{~h}$, symptoms and signs of superinfection of the treated lobe appeared. It was therefore decided to perform a lobectomy in order to minimize the risks of septic shock.

On the third day after the endovascular procedure, the patient underwent thoracotomy with left lower lobectomy, experienced no further blood losses and was hemodynamically stable (Figure $3 a, b$ ).

\section{DISCUSSION}

This case encapsulates the strategy and workflow in treating closed thoracic trauma with major active bleeding in young patients. The first point to keep in mind concerns the fact that a young patient compensates very well immediately post-trauma and then falls abruptly if not treated. Due to the high-energy trauma, this young patient's spine was immediately immobilized and she was transported by helicopter to the nearest trauma center. The second point concerns the execution of a total body CT with contrast (three phases: basal, angio-arterial and venous) which allowed identification and quantification of pulmonary bleeding. The third 


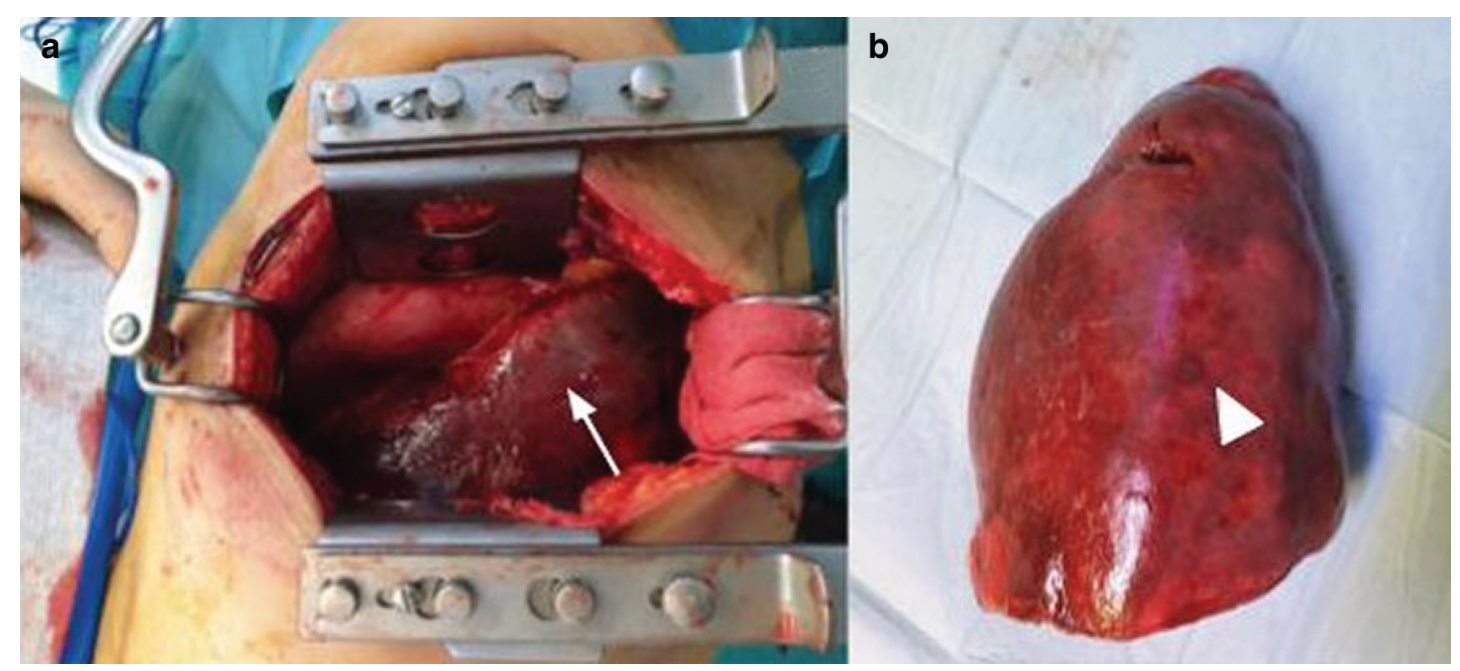

Figure 3 The findings during damage-control thoracotomy and views of the surgical site and operation room just before planned reoperation. (a) The lower lobe of the left lung is remarkably swollen due to intrapulmonary hemorrhage and hematoma (white arrow). (b) The removed lower lobe of the left lung is shown (white arrow head).

point concerns the multidisciplinary decision. Performing an embolization had a double objective: (1) to quickly stabilize the patient who was about to experience hemorrhagic shock, and (2) to try to avoid a lobectomy in such a young patient.

In the literature there are very few reports of embolization of traumatic pulmonary artery bleeding [2], although there are many examples of arteriovenous malformation and pseudoaneurysm embolization. The use of different types of embolizing agents, spirals, glue, non-adhesive liquid embolizing agents (such as ONYX, SQUID, etc.) is described [3]. In our case, the patient showed no alterations in the coagulation profile and, therefore, we opted for the use of metal coils which are easy to use and have a relatively low cost. Unfortunately, the possible superinfection of the treated lobe or lung segment is an expected complication that redirects the patient toward lobectomy. In trauma, this complication is greater in relation to communication between hematoma and airways.

\section{CONCLUSION}

The use of percutaneous embolization can be considered a valid attempt to avoid lobectomy in young patients; however, superinfection is to be considered a frequent complication.

During the current Covid-19 pandemic, the scarce availability of operating theaters can further lead to the use of interventional radiology as a first approach to trauma.

\section{Ethics Statement}

(1) All the authors mentioned in the manuscript have agreed to authorship, read and approved the manuscript, and given consent for submission and subsequent publication of the manuscript.

(2) The authors declare that they have read and abided by the JEVTM statement of ethical standards including rules of informed consent and ethical committee approval as stated in the article.

\section{Funding}

The authors received no financial support for the research, authorship, and/or publication of this article.

\section{REFERENCES}

[1] Pape HC, Zelle B, Lohse R, et al. Evaluation and outcome of patients after polytrauma can patients be recruited for long-term follow-up? Injury. 2006;37(12): 1197-203.

[2] Nagashima F, Inoue S, Ohta M. A patient with severe polytrauma with massive pulmonary contusion and hemorrhage successfully treated with multiple treatment modalities: a case report. J Med Case Rep. 2020; 14(1):69. doi: 10.1186/s13256-020-02406-9. PMID: 32539846; PMCID: PMC729545

[3] Sharma P, Kochar P, Sharma S, et al. A case of pulmonary arteriovenous malformation: role of interventional radiology in diagnosis and treatment. Ann Transl Med. 2017;5(17):345. doi: 10.21037/atm.2017.06.23. PMID: 28936439; PMCID: PMC5599280. 